\title{
Optimization of Corrugated Bulkhead Forms By Genetic Algorithm
}

Joe-Ming Yang

Associate Professor, Department of Naval Architecture and Marine Engineering, National Cheng-Kung University, Tainan, Taiwan, R.O.C., z7908036@email.ncku.edu.tw

Cheng-Neng Hwang

Associate Professor, Department of Naval Architecture and Marine Engineering, National Cheng-Kung University, Tainan, Taiwan, R.O.C.

Follow this and additional works at: https://jmstt.ntou.edu.tw/journal

Part of the Engineering Commons

\section{Recommended Citation}

Yang, Joe-Ming and Hwang, Cheng-Neng (2021) "Optimization of Corrugated Bulkhead Forms By Genetic Algorithm," Journal of Marine Science and Technology. Vol. 10: Iss. 2, Article 10.

DOI: $10.51400 / 2709-6998.2313$

Available at: https://jmstt.ntou.edu.tw/journal/vol10/iss2/10

This Research Article is brought to you for free and open access by Journal of Marine Science and Technology. It has been accepted for inclusion in Journal of Marine Science and Technology by an authorized editor of Journal of Marine Science and Technology. 


\title{
OPTIMIZATION OF CORRUGATED BULKHEAD FORMS BY GENETIC ALGORITHM
}

\author{
Joe-Ming Yang and Cheng-Neng Hwang
}

Key words: genetic algorithms, corrugated bulkheads, minimum cost.

\begin{abstract}
In this research, the optimal design of corrugated bulkheads being used in ship structures is explored by means of the state-of-theart genetic algorithms. The influences of genetic parameters, such as population sizes, crossover probability and mutation probability, on the optimal design of corrugated bulkheads are carefully examined. An objective function defined for the cost of materials and labors is to be minimized to attain the least cost of panel production. The obtained optimal values of each design variable must satisfy the predescribed constraints and some restrictions on fabrication. The optimal values of minimum weight and minimum cost of a panel production are obtained. Finally, a comparison between the method of genetic algorithm and the sequential linear programming optimization algorithm is made to demonstrate the superiority of the proposed genetic algorithms. The numerical simulation results reveal that the genetic algorithms can efficiently minimize panels' weight and costs in production.
\end{abstract}

\section{INTRODUCTION}

Genetic algorithm (GA) searching procedures are fundamentally based on the mechanics of natural section, the natural genetics and the Charles Darwin's principle of survival of the fittest. Genetic algorithms, which possess the merits of mathematics, biology and engineering, are used to solve a wide range of practical problems. A simple genetic algorithm is composed of three basic genetic operators, such as reproduction, crossover and mutation, which are utilized to find the global optimum of design variables in complex space. Since the genetic algorithm can work on coded design variables and search for a population of points simultaneously, it is easy to use this method to obtain the global optimum. Besides, the GA method uses

Paper Submitted 05/03/02, Accepted 11/05/02. Author for Correspondence: Joe-Ming Yang.

*Associate Professor, Department of Naval Architecture and Marine Engineering, National Cheng-Kung University, Tainan, Taiwan, R.O.C. E-mail: z7908036@email.ncku.edu.tw probabilistic transition rules instead of the usual deterministic ones to increase its manifold variety. Furthermore, it can be applied in various fields, such as applied science, engineering, economic science ... etc. $[1,2]$.

Because of the above advantages and superiority of the genetic algorithm method, the genetic algorithm approach is used in this study to search for the optimal design values of corrugated bulkhead. The results of this investigation are then compared to those obtained from the sequential linear programming (SLP) optimization method. In the past, the SLP method was widely used in optimal structural design of stiffened panels with satisfactory results $[3,4]$. Thus, the GA method has recently become a powerful alternative for cost optimization of basic ship structural elements $[2,5,6]$. For example, Kuo and Wang used discrete optimization method with genetic algorithm to obtain the optimization of torsional strength of a container ship's structure [6].

In the present investigation, the optimization of corrugated bulkhead forms using genetic algorithm is studied, and a comparison between the genetic algorithms and the sequential linear programming optimization algorithm is also made under similar loading and constraints for a corrugated plate.

\section{GENETIC ALGORITHMS APPROACH}

\section{Fitness Function}

Fitness function is a deterministic tool to evaluate the fitness of each chromosome. In order to establish fitness function in the genetic algorithm, two basic rules must be satisfied: (1) the calculated fitness value for each individual or string should be positive, and (2) the best individual should have the highest fitness value. The fitness function is the connection between the physical problem being optimized and the genetic algorithm. The fitness function $F(x)$ can be defined as an objective function $f(x)$ in engineering applications. 
In general, the genetic algorithm is used to solve the unconstrainted optimization problems. As for the constrainted optimization problems, a transformation must be made.

An optimal design problem is to minimize the objective function $f(x)$ subjected to constraints that can be described as follows:

$$
\begin{array}{ll}
\min f(x) & \\
h_{j}(x)=0 & j=1,2, \ldots, p \\
g_{j}(x) \leq 0 & j=p+1, \ldots, m \\
x_{i}^{l} \leq x_{i} \leq x_{i}^{u} & i=1, \ldots, n
\end{array}
$$

Where $x=\left\{x_{1}, x_{2}, \ldots, x_{n}\right\}^{T}$ is a vector containing $n$ design variables; $x_{i}^{l}$ and $x_{i}^{u}$ denote the lower limit and the upper limit, respectively; $f(x)$ is the objective function to be minimized; $h_{j}(x)$ and $g_{j}(x)$ are the constraints. In this study, the above constrained minimization problem can be transformed into an unconstrained one by adding a penalty term, which can transform the constrained objective function $f(x)$ to a pseudo objective function $\Phi(x, r)$ :

$$
\Phi(x, r)=f(x)+r\left\{\sum_{j=1}^{p}\left|h_{j}(x)\right|+\sum_{j=p+1}^{m} \max \left[0, g_{j}(x)\right]\right\}
$$

Where $r$ is a penalty coefficient.

The goal of optimization here is to find the minimum of objective function; the objective function can be expressed as:

$$
f_{j}(x)=\Phi_{\max }(x, r)-\Phi_{j}(x, r)
$$

Where

$j$ is the number of individual and equal to population size.

$f_{j}(x)$ is the $j^{\text {th }}$ objective function.

$\Phi_{\max }(x, r)$ denotes the maximum fitness value of the entire population. It is an explicit value for a set of $(x, r)$.

$\Phi_{j}(x, r)$ indicates the $\mathrm{jth}$ fitness value of individual string after adding a penalty term.

\section{Mechanism of Genetic Search}

The mechanism of a genetic algorithm is very simple and it involves nothing other than copying string and swapping partial string. Basically, this search procedure utilizes three operators: reproduction, crossover and mutation to find the best solution. Detailed illustration of these operators can be found in references $[1,2,7]$. Fig. 1 shows the flow chart of a simple genetic algorithm optimizer.

Traditionally, the design variables are coded as binary unsigned integers, which are finite-length strings. However, in this study, real-valued type coding is used to reduce the processing of coding and decoding during manipulating the design variables. In this procedure, the design variables can be treated as a chromosome directly.

\section{OPTIMIZATION MODELS OF SHIP STIFFENED PANELS}

In this investigation, a corrugated bulkhead model representing the typical compartment of existing bulk carriers is selected as an example to demonstrate the feasibility of numerical calculations.

\section{Design variables}

Based on the theoretical and experimental studies on the ultimate strength of corrugated bulkheads by Paik et al. [8], a conclusion is made that a single central corrugation may be taken as representative of the whole corrugated bulkhead. Therefore, four dimensions of a single corrugation, including plate thickness $t_{p}$, web

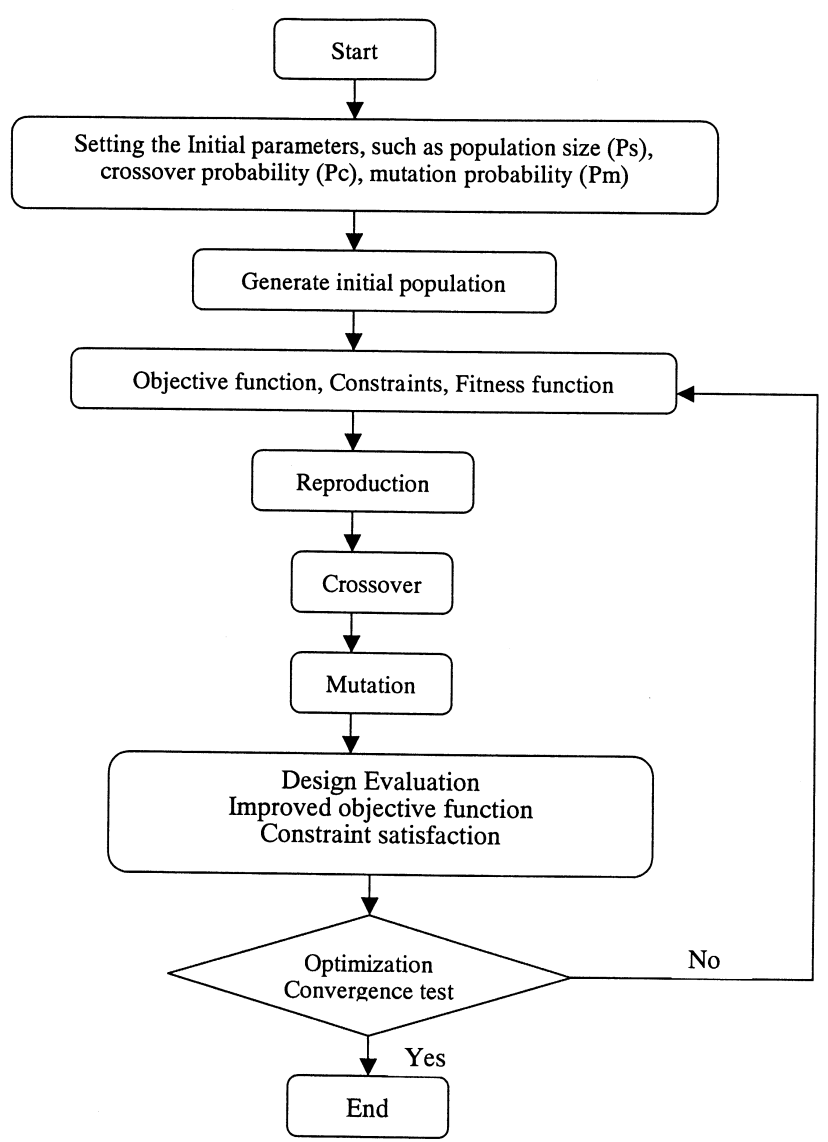

Fig. 1. Flow chart of a simple genetic algorithm optimizer. 
width $c$, depth of corrugation $d$ and number of corrugations $N_{c}$, are selected as design variables, which are shown in Fig. 2, where $B=4123 \mathrm{~mm}$ and $a=2000 \mathrm{~mm}$ represent the breadth and the length of a panel, respectively. The number of corrugation $N_{c}$ is modeled as an integer variable. The elastic modulus and the yield stress of the material are $210000 \mathrm{~N} / \mathrm{mm}^{2}$ and $240 \mathrm{~N} / \mathrm{mm}^{2}$, respectively. The boundaries of the corrugated bulkhead are assumed to be simply supported.

\section{- Single corrugation}

The variables defined in $x_{c}=\left\{t_{p}, d, c, N_{c}\right\}$ represent the design variables of a single corrugation, and the geometrical constraints of these design variables are shown as follows [3]:

$$
\begin{aligned}
& 5.00 \mathrm{~mm} \leq t_{p} \leq 45.00 \mathrm{~mm} \\
& 100.00 \mathrm{~mm} \leq c \leq 400.00 \mathrm{~mm} \\
& 100.00 \mathrm{~mm} \leq d \leq 400.00 \mathrm{~mm} \\
& 0 \leq N_{c} \leq 20 \\
& 50^{\circ} \leq \theta \leq 90^{\circ}
\end{aligned}
$$

The configuration of a single corrugation is shown in Fig. 2 and the relation between $B, P, b, c, \theta$ and $N_{c}$ is described in below:

$$
N_{c}=B / P, P=2 \times(b+c \times \cos (\theta))
$$

Besides, the above design variables must satisfy the following constraints, which are based on some design codes $[9,10]$,

$$
\begin{aligned}
& 40 \leq b / t_{p} \\
& c / t_{p} \leq 100
\end{aligned}
$$

\section{Objective function}

\section{(1) Minimum weight function}

The total weight of a stiffened panel is a simple algebraic sum of the weights on plates and stiffeners. The weight functions of single corrugation is shown below:

$$
W\left(x_{c}\right)=2(b+c) \times t_{p} \times N_{c} \times a \times g
$$

where $\gamma=7.85$ ton $/ \mathrm{m}^{3}$ is the specific weight of mild steel.

\section{(2) Minimum production cost function}

Total production cost includes material cost and labor cost. The material cost is easy to be calculated but the labor cost may be affected by many uncertain factors. A simplified general total cost model including as many production factors as possible is proposed by Rahmam [3] and is expressed as follows:

$$
C_{t o t}\left(x_{c}\right)=P_{a} W\left(x_{c}\right)+N_{c} a P_{s}\left(C_{s 1}+C_{s 2}\right)
$$

Where $P_{a}$ is the material price per unit material weight, $N_{c}$ is the total number of corrugations, $a$ is the span of the panel, $P_{s}$ is the labor rate including overheads (cost/hour), $C_{s 1}$ is the labor hour required per meter welding of stiffeners to plates and $C_{s 2}$ is the labor hour required per meter fabrication of corrugated bulkhead.

In equation (9), by using appropriate values of $N_{c}$, the values of $C_{s 1}, C_{s 2}$ and $C_{t o t}\left(x_{c}\right)$ can be obtained for corrugated bulkhead forms. Besides, $C_{s 1}$ and $C_{s 2}$ are different for different types of panel forms and are varying in different shipyards and countries depending on technical facilities and labor skills. In order to compare our results with those in reference [3], the production condition of shipyard in Bangladesh is considered. The $C_{s 1}$ is assumed to be $1.2 \mathrm{hour} / \mathrm{m}$ and the $C_{s 2}$ can be represented as $C_{s 2}=C_{c}+C_{f}$, where $C_{c}$ is the labor hour required for per meter of plate cutting and $C_{f}$ is the forming time required per meter of corrugation. The value of $C_{c}=0.25 \mathrm{hour} / \mathrm{m}$ and the value of $C_{f}=0.55$ are selected for the present study.

\section{Constraints}

The optimal designs of corrugated bulkhead must be achieved to satisfy the following constraints: ultimate failure constraints and serviceability failure constraints [3]. Detailed definitions of properties of corrugated section and evaluation of various constraint functions are shown in Appendix A and Appendix B, respectively as well as in reference [3].

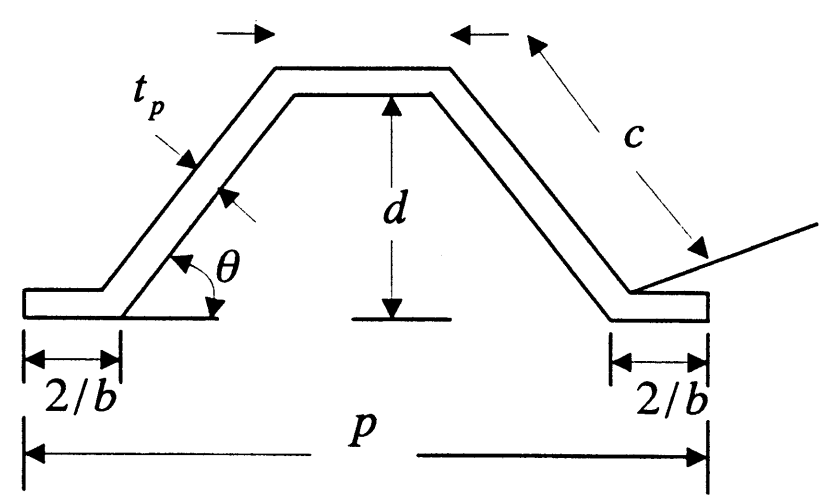

Fig. 2. Configuration of a single corrugation. 


\section{(1) Ultimate failure constraints}

Beam-column collapsed by plate compression, (flange plate)

$$
g_{1}\left(x_{c}\right)=\sigma_{u p}-S F_{u} \times \sigma_{a} \geq 0
$$

Ultimate plate failures of flange plate,

$$
g_{2}\left(x_{c}\right)=\sigma_{u f p}-S F_{u}-\sigma_{a} \geq 0
$$

Ultimate plate failures of web plate,

$$
g_{3}\left(x_{c}\right)=\sigma_{u w p}-S F_{u}-\sigma_{a} \geq 0
$$

\section{(2) Serviceability failure constraints}

Pre-mature plate buckling flange plate,

$$
g_{4}\left(x_{c}\right)=\sigma_{c r f}-S F_{s} \times \sigma_{f} \geq 0
$$

Initial yielding of flange plate,

$$
g_{5}\left(x_{c}\right)=\sigma_{y}-S F_{s} \times \sigma_{f} \geq 0
$$

Pre-mature web instability,

$$
g_{6}\left(x_{c}\right)=\sigma_{c r w}-\sigma_{a} \geq 0
$$

Yielding due to transverse bending of plate,

$$
g_{7}\left(x_{c}\right)=\sigma_{y}-S F_{s} \times \sigma_{\max } \geq 0
$$

Large permanent set of flange plate

$$
g_{8}\left(x_{c}\right)=p_{c r f}-S F_{s} \times p \geq 0
$$

Large permanent set of web plate

$$
g_{9}\left(x_{c}\right)=p_{c r w}-S F_{s} \times p \geq 0
$$

Where $S F_{u}$ and $S F_{s}$ are equal to 1.6 and 1.4 , respectively.

\section{RESULTS AND DISCUSSIONS}

In this study, a genetic algorithm program, named Evolver, which can handle the continuous and discrete design variables, is used to find optimal corrugated bulkheads.

There are many factors that affect the results of genetic algorithms in finding optimal values. In this investigation, the effects of population size (PS), probability of crossover $(\mathrm{Pc})$ and probability of mutation (Pm) are studied for corrugated bulkhead. The optimal values of presented study are then compared with those of Rahman's study, which was done by the Sequential Linear Programming (SLP) technique.

Based on the studies made by Goldberg [1] and by $\mathrm{Wu}$ et al. [5], the higher probability of crossover and lower probability of mutation are suggested. Therefore, $P c=0.8$ and $P m=0.1$ are selected as initial values in this study.

\section{Optimization on corrugated bulkhead}

With $P c=0.8, P m=0.1$ and population sizes (PS) ranging from 30 to 200 , trials are made to investigate the effects of generation sizes on the optimal solution. The optimal results are listed in Table 1 as well as in the results of Rahman's study [3]. Table 1 shows that the final total cost values for $P S=60$ to 170 become steady and are almost the same. It is found that the $P S=130$ case has the lowest total cost value and the $P S=80$ case obtains the second lowest total cost value.

In the second step, seven trails with $P S=130, \mathrm{Pm}$ $=0.1$ and $P c=0.5-0.9$ are carried out. All of the results shown in Table 2 are lower than those of Rahman's results. The lowest total cost value is found in $P c=0$. 8 case. Finally, trails are made for $P S=90, P c=0.8$ and $P m=0.001$ to 0.3 cases and for $P S=120, P c=0.9$ and $P m=0.001$ to 0.3 cases to find the influence of mutation. The results are shown in Table 3. It is found that with too small mutation probabilities, the solution becomes worse. The best solution is found in the $P m=0.1$ case, in which the lowest cost value is obtained as Ctot = $12161 \$$ shown in Table 3.

\section{Discussions}

Based on above numerical study, it shows that a trial with $P s=130, P m=0.1, P c=0.8$ gives the best result. The results listed in reference [6] also show that a trial with $P c=0.85$ and $P m 0.1$ gives better results than a trial with $P c=0.9$ and $P m=0.01$. It may be concluded that in general the probability of crossover is much higher than probability of mutation except the special case in reference [11].

Reference [6] has concluded that the higher population sizes (Ps) give the better results. However, in present study, the cases with population sizes higher than 180 or lower than 40 do not give good results. Different conclusions are drawn in evaluation of population size between the present investigation and the reference [6]. This may attributed to the fact that there are 18 cases that are evaluated in the present study, but only three cases $(P s=20,40,60)$ were studied in reference [6].

It is found that no matter how we choose the 
Table 1. Optimum results between GA and SLP [3] for $P c=0.8$ and $P m=0.1$ fixed

\begin{tabular}{lcccccccc}
\hline$[3]$ & 6.67 & 301.73 & 341.83 & 267.14 & 51.4 & 4 & 0.54 & 15934 \\
\hline Ps & $t_{p}$ & $b$ & $c$ & $d$ & $\theta$ & $N_{c}$ & Weight & Min cost \\
Initial & 15.00 & 300.00 & 300.00 & 229.81 & 50.00 & 6.87 & 1.9405 & 52715 \\
value & & & & & & & \\
30 & 5.62 & 363.08 & 236.9 & 181.77 & 50.11 & 4 & 0.42331 & 13031 \\
40 & 5.62 & 362.89 & 236.85 & 181.55 & 50.04 & 4 & 0.42314 & 13027 \\
50 & 5.53 & 359.63 & 241.56 & 185.16 & 50.04 & 4 & 0.41790 & 12896 \\
60 & 5.29 & 351.78 & 255.67 & 196.79 & 50.33 & 4 & 0.40369 & 12540 \\
70 & 5.25 & 350.48 & 258.53 & 199.43 & 50.48 & 4 & 0.40126 & 12480 \\
80 & 5.2 & 347.39 & 260.93 & 199.98 & 50.03 & 4 & 0.39707 & 12375 \\
90 & 5.52 & 359.47 & 242.13 & 185.58 & 50.03 & 4 & 0.41725 & 12879 \\
100 & 5.75 & 367.66 & 229.93 & 176.51 & 50.14 & 4 & 0.43131 & 13213 \\
110 & 5.45 & 357.26 & 246.15 & 188.97 & 50.15 & 4 & 0.41323 & 12779 \\
120 & 5.34 & 353.16 & 252.57 & 193.91 & 50.15 & 4 & 0.40633 & 12606 \\
130 & 5.06 & 342.22 & 269.63 & 207 & 50.15 & 4 & 0.38850 & 12161 \\
140 & 5.05 & 282.35 & 201.8 & 154.65 & 50.03 & 4 & 0.38349 & 12647 \\
150 & 5.42 & 355.92 & 248.26 & 190.6 & 50.15 & 4 & 0.41096 & 12722 \\
160 & 5.43 & 356.6 & 247.32 & 189.94 & 50.17 & 4 & 0.41206 & 12750 \\
170 & 5.19 & 322.86 & 300 & 230.4 & 50.17 & 4 & 0.40584 & 12594 \\
180 & 5.58 & 354.4 & 239.75 & 186.55 & 51.09 & 4 & 0.42344 & 13034 \\
190 & 5.75 & 371.18 & 230.44 & 180.06 & 51.38 & 4 & 0.43473 & 13316 \\
200 & 5.77 & 368.39 & 230.64 & 178.05 & 50.53 & 4 & 0.43382 & 13294 \\
\hline
\end{tabular}

Table 2. Optimum results between GA and SLP [3] for $P S=130$ and $P m=0.1$ fixed

\begin{tabular}{lcccccccc}
\hline$[3]$ & 6.67 & 301.73 & 341.83 & 267.14 & 51.4 & 4 & 0.54 & 15934 \\
\hline Pc & $t_{p}$ & $b$ & $c$ & $d$ & $\theta$ & $N_{c}$ & Weight & Min cost \\
Initial & 15.00 & 300.00 & 300.00 & 300.00 & 90.00 & 6.87 & 1.9405 & 52715 \\
value & & & & & & & 0.39015 & 12202 \\
0.5 & 5.06 & 343.85 & 270.09 & 208.95 & 50.68 & 4 & 0.39966 & 12423 \\
0.55 & 5.23 & 348.64 & 259.29 & 198.88 & 50.09 & 4 & 0.43160 & 13238 \\
0.6 & 5.75 & 367.72 & 229.97 & 176.63 & 50.18 & 4 & 0.42027 & 12955 \\
0.65 & 5.55 & 362.06 & 241.38 & 186.75 & 50.68 & 4 & 41922 & 12929 \\
0.7 & 5.55 & 360.66 & 240.44 & 184.36 & 50.06 & 4 & 0.41922 & 12413 \\
0.75 & 5.21 & 348.71 & 260.66 & 200.72 & 50.36 & 4 & 0.39859 & 12161 \\
0.8 & 5.06 & 342.22 & 269.63 & 207 & 50.15 & 4 & 0.38850 & 12587 \\
0.85 & 5.02 & 283.37 & 200.61 & 153.94 & 50.12 & 4 & 0.38108 & 0.43073 \\
0.9 & 5.66 & 369.65 & 235.79 & 185.66 & 51.94 & 4 & & 13216 \\
\hline
\end{tabular}

Table 3. Optimum results between GA and SLP [3] for $P s=130$ and $P c=0.8$ fixed

\begin{tabular}{lcccccccc}
\hline$[3]$ & 6.67 & 301.73 & 341.83 & 267.14 & 51.4 & 4 & 0.54 & 15934 \\
\hline Pm & $t_{p}$ & $b$ & $c$ & $d$ & $\theta$ & $N_{c}$ & Weight & Min cost \\
Initial & 15.00 & 300.00 & 300.00 & 300.00 & 90.00 & 6.87 & 1.9405 & 52715 \\
value & & & & & & & \\
0.01 & 5.07 & 281.96 & 202.7 & 155.5 & 50.09 & 5 & 0.38560 & 12700 \\
0.05 & 5.15 & 279.89 & 205.91 & 157.95 & 50.09 & 5 & 0.39262 & 12876 \\
0.1 & 5.06 & 342.22 & 269.63 & 207 & 50.14 & 4 & 0.38850 & 12161 \\
0.15 & 5.28 & 351.74 & 256.22 & 197.47 & 50.42 & 4 & 0.40345 & 12534 \\
0.2 & 5.72 & 369.55 & 232.32 & 181.15 & 51.24 & 4 & 0.43211 & 13251 \\
0.25 & 5.1 & 281.14 & 203.86 & 156.32 & 50.07 & 5 & 0.38807 & 12762 \\
0.3 & 5.09 & 281.79 & 203.68 & 156.63 & 50.26 & 5 & 0.38811 & 12763 \\
0.4 & 5.71 & 366.65 & 231.93 & 178.28 & 50.24 & 4 & 0.42931 & 13181 \\
0.5 & 5.44 & 359.7 & 247.92 & 193.24 & 51.21 & 4 & 0.41531 & 12831 \\
\hline
\end{tabular}


Table 4. The constrained results of present study and reference [3]

\begin{tabular}{lccccccccc}
\hline Constraints & $g_{1}$ & $g_{2}$ & $g_{3}$ & $g_{4}$ & $g_{5}$ & $g_{6}$ & $g_{7}$ & $g_{8}$ & $g_{9}$ \\
\hline Present study & 287.14 & 34.37 & 77.40 & 100.64 & 101.64 & 80.51 & 26.65 & 0.09 & 0.20 \\
Reference [3] & 1055.40 & 98.78 & 83.16 & 108.16 & 109.15 & 84.21 & 108.15 & 0.33 & 0.24 \\
\hline
\end{tabular}

population sizes, the value of crossover or mutation, the results obtained by genetic algorithms are better than those obtained from the SLP method. This may attribute to the fact that the results obtained by the GA method satisfy all the constraints shown in Eqs. (10) to (17), and the values are close to the bounds of the constraints, while the results obtained from the SLP method are usually much higher than the boundary requirements of those constraints though they also meet those constraints, which are shown in Table 4. In Table 4, all the results of present study are lower than those made in reference [3]. It is also found that the optimal result of the minimum cost value does not necessitate having the minimum weight.

As stated in the beginning, the genetic algorithm searches for a population of points at a time, not for a single point. Although, in some cases, the genetic algorithm method searches for more than hundred points at a time, it takes time less than one minute to finish a search job in this study. The results of the SLP method are mainly dependent on the selection of initial values. If a lot of continuous and discrete design variables are involved in an optimization searching problem, the SLP method may fall in finding the local optimum.

\section{CONCLUSIONS}

Based on the above numerical investigation, the following observations or conclusions are made:

- The GA searching technique provides an alternative method for structural optimization. With appropriate population size, crossover probability and mutation probability, the GA searching procedure yields fine results.

- It is found that both plate thickness $t_{p}$ and total number of corrugation $N_{c}$ have strong influence on the material weight function $W\left(x_{c}\right)$ in the optimization process while the number of corrugation $N_{c}$ and the corrugation angle $\theta$ have considerable contribution on the total production cost $C_{t o t}\left(x_{c}\right)$.

- Based on the results shown in Table 2 and Table 3 , it is found that the effects of crossover and mutation are much less than what we expected. The difference percentage between the best result $\left(C_{t o t}\left(x_{c}\right)=12161, P c\right.$ $=0.8)$ and the worst result in both Table 2 and Table 3 is less than $9 \%$.

\section{REFERENCES}

1. Goldberg, D.E., "Genetic Algorithms in Search, Optimization and Machine Learning," Addison-Wesley Publishing Company, Inc. (1989).

2. Nobukawa, H. and Zhou, G., "Discrete Optimization of Ship Structures with Genetic Algorithms," Journal of The Society of Naval Architects of Japan, Vol. 179, pp. 293-301 (1996).

3. Rahman, M.K., "Optimization of Panel Forms for Improvement in Ship Structures," Structural Optimization, Vol. 11, pp. 195-212 (1996).

4. Leheta, H.W. and Mansour, A.E., "Reliability-based Method for Optimal Structural Design of Stiffened Panels," Marine Structures, Vol. 10, pp. 323-352 (1997).

5. Wu, J. and Xu, C., "Ship Structural Optimum Design With Mix-discrete Variables via the Genetic Algorithm," written in Chinese, Shipbuilding of China, No. 140, pp. 57-65 (1998).

6. Kuo, H.C. and Wang, H.G., "Discrete Optimization of Torsional Strength for Containership Structures by Using Genetic Algorithms", Journal of the Society of Naval Architects and Marine Engineers, R.O.C., Vol. 18, No. 1, pp. 47-56 (1999).

7. Johnson, J.M. and Yahya, R.S., "Genetic algorithms in Engineering Electromagnetics," IEEE Antennas and Propagation Magazine, Vol. 39, No. 4 (1997).

8. Paik, J.M. Thayamballi, A.K., and Chun, M.S., "Theoretical and Experimental Study on the Ultimate Strength of Corrugated Bulkheads," Journal of Ship Research, Vol. 41, No. 4, pp. 301-317 (1997).

9. Lloyds' Register of Shipping, Small Ships; Rules for the hull construction of steel ships under $90 \mathrm{~m}$ in length (1976).

10. Nelson, H.M., Wright, D.T. and Dolphin, J.W., "Demonstrations of Plastic Behavior of Steel Frames," ASCE, Vol.83, No. EM4 (1957).

11. Gen, M. and Cheng, R., "Genetic Algorithms and Engineering Design,” John Wiley \& Sons, Inc. (1997).

\section{Appendix A: Properties of corrugated sections [3]:}

$Z=\frac{1}{3} t_{p} d(3 b+c)$ : is the elastic section modulus ( $d$ is the height of corrugation; $t_{p}, \mathrm{~b}$ and $\mathrm{c}$

$Z_{p}=\frac{1}{2} t_{p} d(2 b+c):$ is the plastic section modulus. 
$I=\frac{1}{6} t_{p} d^{2}(3 b+c):$ is the moment of inertia about neutral axis.

$A_{s}=2(b+c) t_{p}: \quad$ is the cross-sectional area of one $y_{f}=\frac{d}{2}:$ is the distance of flange from neutral axis.

\section{Appendix B: Constraints of corrugated sections}

\section{- Ultimate failure constraints[3]}

(1) Beam-column collapse by flange plate failure:

$$
\begin{aligned}
& g_{1}\left(x_{c}\right)=\sigma_{u p}-S F_{u} \times \sigma_{a} \geq 0 \\
& \sigma_{u p}=\sigma_{u e} \frac{A_{e}}{A} \quad b_{e}=b \times T \\
& T=\frac{E_{s}}{E}=0.25\left(2+\xi-\sqrt{\xi^{2}-\frac{10.4}{\beta^{2}}}\right) \quad \xi=1+\frac{2.75}{\beta^{2}} \\
& \beta=\frac{b}{t_{p}} \times \sqrt{\frac{\sigma_{y}}{E}} \quad \Delta_{p}=h \times A_{s} \times\left(\frac{1}{A_{s}}-\frac{1}{A}\right)
\end{aligned}
$$

$\sigma_{a}:$ the axial stresses, in $\mathrm{N} / \mathrm{mm}^{2}$.

$S F_{u}, S F_{s}$ : the safety factors against ultimate and serviceability failures, respectively. Their respective values, which have been used for designs presented in this paper, are 1.6 and 1.4 .

$A_{e}, I_{e}:$ effective area and effective moment of inertia

$h$ : the distance between the mid-thickness of the plate

flange and the centroidal axis of the cross-section $A_{s}$ : cross sectional area of stiffener

$$
\begin{aligned}
& \sigma_{F}=\frac{\sigma_{Y}(T-0.1)}{T}, \quad \sigma_{u e}=\sigma_{F} \times R \quad \mu=\frac{M_{0} y_{p}}{I_{e} \sigma_{F}} \\
& R=\frac{\zeta}{2}-\sqrt{\frac{\zeta^{2}}{4}-\frac{1-\mu}{\left(1+\eta_{p}\right) \lambda^{2}}} \quad \eta_{p}=\frac{\Delta_{p} y_{p}}{\rho_{e}^{2}} \\
& \zeta=\frac{1-\mu}{1+\eta_{p}}+\frac{1-\eta_{p}+\eta}{\left(1+\eta_{p}\right) \lambda^{2}} \quad \eta=\frac{\left(\delta_{0}+\Delta\right) y_{p}}{\rho_{e}^{2}} \\
& \lambda=\frac{a}{\pi \rho_{e}} \sqrt{\frac{\sigma_{F}}{E}}
\end{aligned}
$$

$y_{p}$ The distance between the mid-thickness of the plate flange and the centroidal axis of the effective cross-section

$$
M_{0}=\frac{p b a^{2}}{8} \quad \delta_{0}=\frac{5 p b a^{4}}{384 E I} \quad \Delta=\frac{a}{750}
$$

(2) Ultimate failure of flange and web plates:

$g_{2}\left(x_{c}\right)=\sigma_{u f p}-S F_{s} \times \sigma_{a} \geq 0, g_{3}\left(x_{c}\right)=\sigma_{u w p}-S F_{s} \times \sigma_{a} \geq$ 0 $\sigma_{\text {upl }}= \begin{cases}\sigma_{y} \cdot\left(\frac{2.5}{\beta}-\frac{1.5625}{\beta^{2}}\right) \cdot R_{r}, & \beta \geq 1.25 \\ \sigma_{y} \cdot R_{r} & , \quad 0 \leq \beta<1.25\end{cases}$

$\beta=\frac{b}{t_{p}} \cdot \sqrt{\frac{\sigma_{y}}{E}}$

$R_{r}= \begin{cases}1-\frac{\sigma_{r}}{\sigma_{y}} \cdot \frac{E_{t}}{E} \cdot \frac{\beta}{2.5 \beta-1.5625} & , \quad \beta \geq 1.25 \\ 1-\frac{\sigma_{r}}{\sigma_{y}} \cdot \frac{E_{t}}{E} & , 0 \leq \beta<1.25\end{cases}$

$\frac{E_{t}}{E}= \begin{cases}{\left[\frac{6.31 \beta^{2}}{39.8+P_{r} \cdot\left(1-P_{r}\right) \cdot \beta^{2}}\right]^{2},} & 0 \leq \beta \leq \frac{2.51}{\sqrt{P_{r}}} \\ 1.0 \quad, \beta>\frac{2.51}{\sqrt{P_{r}}}\end{cases}$

$\sigma_{r}=\frac{2 \eta \cdot \sigma_{y}}{\frac{b}{t_{p}}-2 \eta} ; \quad \eta=4.5 \sim 6 \quad P_{r}=0.5$

\section{- Serviceability failure constraints:}

(1) Local buckling and initial yielding of flange plate:

$\geq 0$

$g_{4}\left(x_{c}\right)=\sigma_{c r f}-S F_{s} \times \sigma_{f} \geq 0, g_{5}\left(x_{c}\right)=\sigma_{y}-S F_{s} \times \sigma_{f}$

$\sigma_{c r e f}=\frac{k_{\min } \pi^{2} E}{12\left(1-v^{2}\right)}\left(\frac{t_{p}}{b}\right)^{2} \quad \sigma_{f}=\sigma_{a}+\frac{M_{0} y_{f}}{I}$

$k_{\min }= \begin{cases}-2 \frac{c}{b}+6, & 0 \leq \frac{c}{b} \leq 1 \\ -3 \frac{c}{b}+7, & 1<\frac{c}{b} \leq 1.75 \\ -\frac{c}{b}+3.5, & \frac{c}{b}>1.75\end{cases}$

(2) Local instability of web plate:

$g_{6}\left(x_{c}\right)=\sigma_{c r w}-\sigma_{a} \geq 0$

$k_{\min }= \begin{cases}-2 \frac{c}{b}+6, & 0 \leq \frac{c}{b} \leq 1 \\ -3 \frac{c}{b}+7, & 1<\frac{c}{b} \leq 1.75 \\ -\frac{c}{b}+3.5, & \frac{c}{b}>1.75\end{cases}$

$k_{b}= \begin{cases}13.5 \frac{b}{c}, & 0 \leq \frac{b}{c} \leq 0.5 \\ 1.5 \frac{b}{c}+6, & 0.5<\frac{b}{c} \leq 1.0 \\ 0.1 \frac{b}{c}+7.4, & \frac{b}{c}>1.0\end{cases}$ 
$M_{c r}=\frac{I \cdot \sigma_{c r}}{y_{f}} \quad \sigma_{c r}=\frac{k_{b}^{2} \cdot D}{t_{p} \cdot b^{2}} \quad D=\frac{E \cdot t_{p}}{12\left(1-v^{2}\right)}$

(3) Transverse yielding of flange plate:

$$
\begin{aligned}
& g_{7}\left(x_{c}\right)=\sigma_{y}-S F_{s} \times \sigma_{\max } \geq 0 \\
& \sigma_{\max }=\frac{p}{2} \cdot\left(\frac{b}{t_{p}}\right)^{2} \cdot\left(1-\frac{c}{b}+\frac{c^{2}}{b^{2}}\right)
\end{aligned}
$$

(4) Large permanent set of flange and web plates: $\geq 0$ $g_{8}\left(x_{c}\right)=p_{c r f}-S F_{s} \times p \geq 0 \quad g_{9}\left(x_{c}\right)=p_{c r w}-S F_{s} \times p$

$$
\begin{aligned}
& p_{c r}=\frac{Q \sigma_{y}^{2}}{E} \quad Q=Q_{y}+T\left(R_{w}\right) \times\left(\Delta Q_{0}+\Delta Q_{1} \times R_{w}\right) \\
& Q_{y}=\frac{2}{\sqrt{1-v+v^{2}} \times \beta^{2}} \times\left[1+0.6 \times\left(\frac{b}{a}\right)^{4}\right]
\end{aligned}
$$

$$
\Delta Q_{1}=0.32 \times\left[\frac{\left(\frac{b}{a}\right)}{\sqrt{\beta}}\right]^{1.5}
$$$$
Q_{0}=\frac{1+0.5 \times \beta \times\left(\frac{b}{a}\right)\left[1+\left(\frac{b}{a}\right) \times\left(3.3-\frac{1}{\beta}\right)^{4}\right]}{\sqrt{1-v+v^{2}} \times \beta^{2}}
$$$$
R_{w}=\frac{w_{p t}-w_{p i}}{w_{p 0}} \quad w_{p i}=\frac{b}{100} \quad w_{p i}=0.65 \times t_{p} \times \beta^{1.65}
$$$$
w_{p 0}=\frac{0.07 \times \beta^{2} \times t_{p}}{3}
$$

$T\left(R_{w}\right)= \begin{cases}{\left[1-\left(1-R_{w}\right)^{3}\right]^{1 / 3}} & R_{w} \leq 1 \\ 1 & R_{w} \geq 1\end{cases}$ 